\title{
Churg-Strauss Syndrome Concomitant with Chronic Symmetrical Dacryoadenitis Suggesting Mikulicz's Disease
}

\author{
Yusuke Hanioka ${ }^{1}$, Keiko Yamagami ${ }^{1}$, Katsunobu Yoshioka ${ }^{2}$, Tomomi Nakamura ${ }^{1}$, \\ Masatsugu Kishida ${ }^{1}$, Tomoyuki Nakamura ${ }^{1}$, Toshimasa Yamaguchi ${ }^{1}$, Naomi Koshimo ${ }^{3}$, \\ Takeshi Inoue $^{4}$ and Masahito Imanishi ${ }^{1}$
}

\begin{abstract}
A case of Churg-Strauss syndrome complicated by chronic symmetrical dacryoadenitis suggestive of Mikulicz's disease is herein presented. A 72-year-old Japanese man, who had been previously diagnosed with asthma, presented with weakness of the left leg and purpura on the lower extremities. A neurological examination showed multiple mononeuropathies and a laboratory examination revealed elevated eosinophil counts, IgE levels and the presence of Myeloperoxidase-antineutrophil cytoplasmic antibody (MPO-ANCAs). ChurgStrauss syndrome was diagnosed, although the patient also exhibited bilateral swelling of the lachrymal glands. Furthermore, elevated serum IgG4 levels, an infiltration of a relatively large number of IgG4-positive plasmacytes in the nasal mucosa and hypocomplementemia were also observed. These findings were consistent with a diagnosis of Mikulicz's disease (MD). Oral prednisolone (30 mg) was administered and the swelling of the lachrymal glands resolved. Churg-Strauss syndrome may be accompanied by Mikulicz's disease (an IgG4-related disease), and common pathogeneses between Churg-Strauss syndrome and IgG4-related disease may exist.
\end{abstract}

Key words: Churg-Strauss syndrome, Mikulicz's disease, IgG4-related disease

(Intern Med 51: 2457-2461, 2012)

(DOI: 10.2169/internalmedicine.51.7725)

\section{Introduction}

IgG4-related disease (IgG4-RD) was first reported to be autoimmune pancreatitis and then was established to be a systemic disorder characterized by high levels of serum IgG4 and massive fibrosis consisting of highly IgG4-positive plasmacytes infiltrating into various organs (1). A diagnosis of IgG4-RD is not straightforward, as some known pathological conditions meet the same criteria and may be diagnosed as IgG4-RD "in a wide sense." Yamamoto et al. claimed that in order to diagnosis IgG4-RD "in a narrow sense," both swelling of the involved organs and a lack of systemic inflammation must be present (2). Because the definition of IgG4-RD has not yet been established, deter- mining the correct criteria for diagnosing IgG4-RD is difficult. In this study, we defined IgG4-RD in the "narrow sense."

IgG4-RD includes various diseases such as autoimmune pancreatitis, retroperitoneal fibrosis, chronic periaortitis and Kuttner's tumor. Mikulicz's disease (MD), also a component of $\mathrm{IgG4}-\mathrm{RD}$, is characterized by symmetrical and persistent enlargement of the lachrymal and salivary glands and hyposecretion of tears and saliva.

Churg-Strauss syndrome (CSS) is a systemic vasculitis of the small and medium vessels and is associated with extravascular eosinophilic granulomas, peripheral eosinophilia and asthma. Recently, Yamamoto et al. reported that high levels of serum IgG4 and tissue involvement of IgG4positive plasmacytes are seen in patients with CSS (2).

\footnotetext{
${ }^{1}$ Department of Internal Medicine, Osaka City General Hospital, Japan, ${ }^{2}$ Department of Internal Medicine, Osaka City Sumiyoshi Hospital, Japan, ${ }^{3}$ Department of Otorhinolaryngology, Osaka City General Hospital, Japan and ${ }^{4}$ Department of Pathology, Osaka City General Hospital, Japan

Received for publication March 13, 2012; Accepted for publication June 5, 2012

Correspondence to Dr. Yusuke Hanioka, la_sa_sya@ hotmail.com
} 


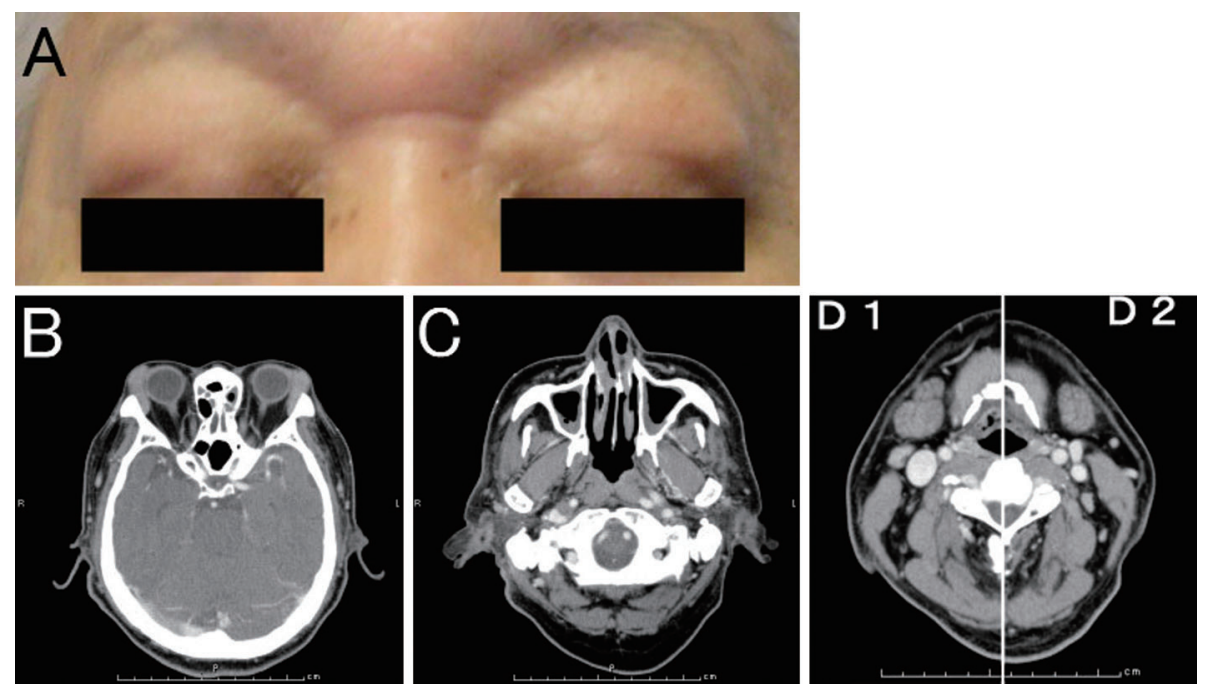

Figure 1. A: Enlarged lachrymal glands. Swelling of the lachrymal glands was without tenderness or erythema. B and C: Contrast-enhanced CT of the head. Slice B displays bilateral swollen lachrymal glands. Slice $C$ : displays thickening of the nasal mucosa and mucous membranes of the maxillary sinus. Slices D1 and D2: show the mild swelling in both the submandibular glands. The swelling was diminished by administration of oral glucocorticoids.

formed. In addition to indicating bilateral swelling of the lachrymal glands, head and neck CT showed mild swelling of the submandibular glands bilaterally, accumulation of fluid in both maxillary sinuses and thickening of the nasal mucosa (Fig. 1B, 1C, 1D). No other particular findings were observed on whole-body CT. Tests for serum anti-nuclear, anti-SS-A, anti-SS-B and anti-double-stranded DNA were negative. The serum IgG4 and IgG levels were significantly elevated at $343 \mathrm{mg} / \mathrm{dL}$ and 2,093 mg/dL, respectively. Hypocomplementemia was indicated by the following data: $\mathrm{C} 3$ : $49.1 \mathrm{mg} / \mathrm{dL} ; \mathrm{C} 4: 4.5 \mathrm{mg} / \mathrm{dL}$; and CH50: $16.9 \mathrm{IU} / \mathrm{mL}$ (Table b).

Hypocomplementemia is an atypical finding in patients with CSS. The patient was also an elderly man who was strongly suspected to have MD. A lachrymal gland biopsy was considered for further study; however, it was not performed due to technical problems. Takano et al. has reported findings from nasal biopsies of MD patients. They found the nasal mucosa of MD patients with olfactory dysfunction to contain infiltrates of numerous IgG4-positive plasmacytes (6). Because of this report, a nasal mucosa biopsy was performed in the present case. An abnormally high number of IgG4-positive plasmacytes and eosinophils were found to be infiltrating the nasal mucosa; however, the number did not reach the cut-off level reported in the literature (Fig. 2).

Therapy with oral prednisolone (30 mg daily) was started. The lachrymal gland swelling improved quickly and the serum complement levels returned to the normal range. The patient's neurological symptoms did not improve dramatically; therefore, intravenous methylprednisolone (1,000 mg) and immunoglobulin infusion therapy were initiated. The patient's symptoms thereafter slowly improved. The patient was discharged after 67 days of hospitalization.

Discussion

The present case was diagnosed as CSS because the patient had multiple mononeuropathies, a history of asthma, paranasal sinus abnormalities, eosinophilia and high levels of serum IgE. In addition, extravascular eosinophils were noted on a skin biopsy and the patient was positive for MPO-ANCAs. In the present case, the serum IgG4 level was significantly elevated and the nasal mucosa biopsy specimen showed infiltration of numerous IgG4-positive plasmacytes. Recently, Yamamoto et al. reported a high level of serum IgG4 and tissue involvement of IgG4-positive plasmacytes to be seen in patients with CSS (2). Therefore, it is possible that CSS can be included as a component of IgG4RD. However, CSS is essentially systemic vasculitis with elements that are different from those of IgG4-RD. In fact, the presence of mononeuropathies is not characteristic of IgG4-RD. Therefore, it is unreasonable to conclude that all of the clinical symptoms observed in the present case result from IgG4-RD or that CSS should not be included in IgG4RD. The patient in the present case exhibited bilateral lachrymal gland swelling. Although CSS is a systemic vasculitis, it does not present with swelling of the affected organs. Therefore, it is also unreasonable to conclude that all of the clinical symptoms observed in the present case are the result of CSS.

Furthermore, the present case included uncommon findings for CSS, such as hypocomplementemia. No previous reports have described the serum complement levels of CSS in detail; however, no review has shown hypocomplementemia in CSS until now. The five cases of CSS described by Yamamoto et al. also showed normal or high levels of CH50 (2). In patients with IgG4-RD, including those with 


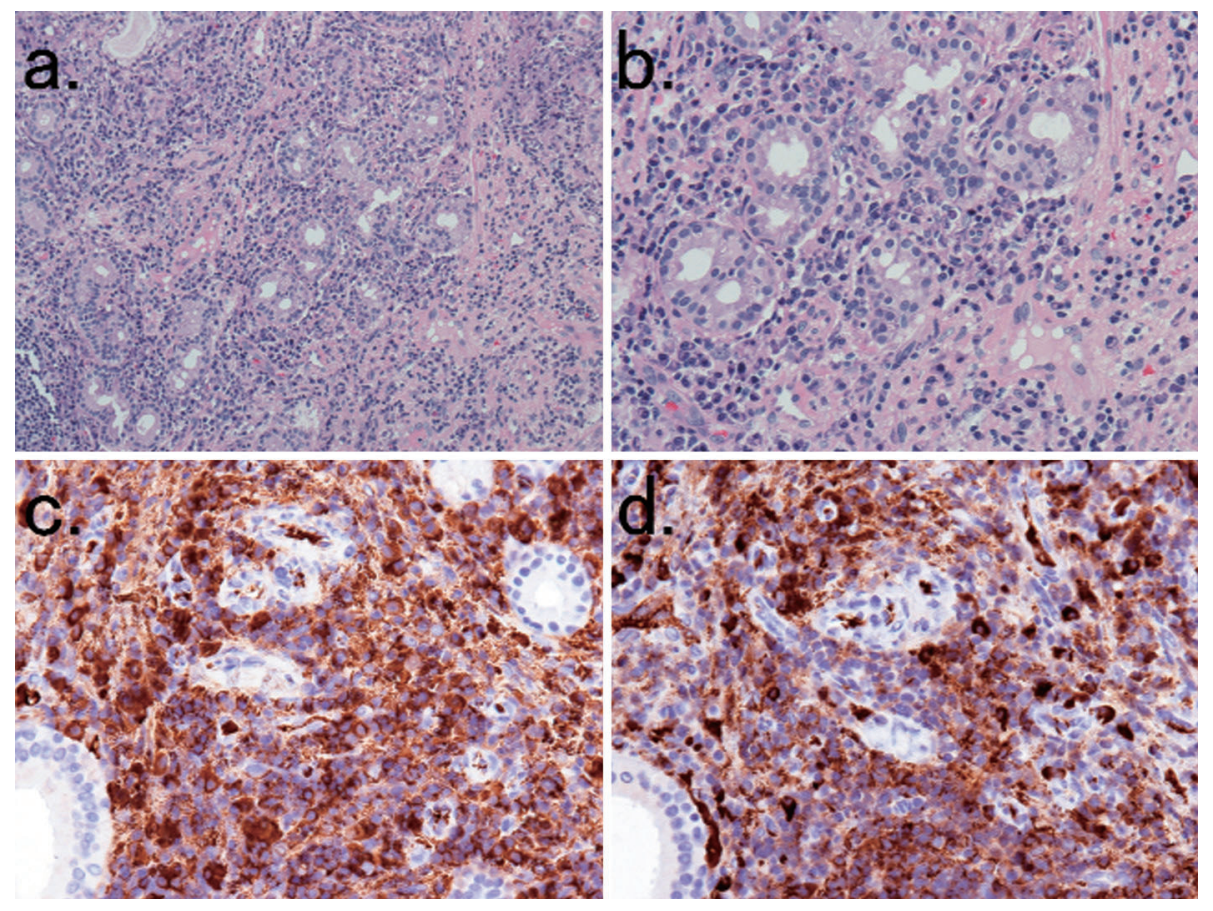

Figure 2. Histopathological findings of the nasal mucosa specimens. a and b, Haematoxylin and Eosin staining shows massive infiltration of lymphocytes and plasmacytes with abundant fibrosis (a, $\times 200 ; \mathrm{b}, \times 400)$. $\mathrm{c}$ and $\mathrm{d}$, Immunohistological staining with anti-IgG and anti-IgG4 antibodies $(\times 400)$. There is a relatively large number of IgG4 positive plasmacytes. The IgG4/IgG ratio is about $40 \%$ in this field.

$\mathrm{MD}$, the serum levels of complement are frequently at low levels and reflect the disease activity (7). The present case showed significantly low levels of $\mathrm{C} 3, \mathrm{C} 4$ and CH50. Hypocomplementemia can occur in patients with systemic lupus erythematous and several other diseases, except for IgG4RD. However, the clinicopathological findings in the present case did not match those of systemic lupus erythematous or other diseases characterized by hypocomplementemia. Therefore, a concomitant diagnosis of IgG4-RD was more likely. A diagnosis of MD (IgG4-RD) was confirmed in the present study.

MD is characterized by symmetric chronic inflammation of the lachrymal and salivary glands. The histology is characterized by lymphoplasmacytic inflammation with numerous IgG4-positive plasmacytes and exuberant fibrosis. Additionally, MD is included in IgG4-RD. Although performing a lachrymal gland biopsy was impossible in the present case because of technical problems, a biopsy of the nasal mucosa showed infiltration of many IgG4-positive plasmacytes. In addition to showing lachrymal enlargement, the patient also exhibited bilateral mildly enlarged submandibular glands (as noted by a radiologist). The size of the submandibular glands reduced after steroid therapy was administered. Although histological confirmation was not obtained, it was determined that the patient fulfilled the Clinical Diagnostic Criteria of IgG4-Related Mikulicz's Disease advocated by the Japanese Medical Society for Sjogren's Syndrome (8). A limitation to the present study includes the fact that the size of the submandibular glands may be not clinically signifi- cant; however, a final diagnosis of chronic sclerosing dacryoadenitis (a subset of IgG4-RD) was made (9).

Both $\mathrm{IgG} 4$ and IgE are produced as a result of Th2 cytokines (for example, IL-4, IL-5 and IL-10), and IgG4 is considered to be derived from an IgE-blocking antibody. The mechanisms underlying the elevation of $\operatorname{IgE}$ and the association between high levels of serum IgE and elevated serum IgG4 levels seen in patients with IgG4-RD are wellunderstood. Although CSS differs completely from MD, they share common characteristics: both diseases develop in patients with allergic symptoms such as asthma or atopic dermatitis. This is evidence for the existence of a similar pathological foundation involving the actions of Th2 cells in both CSS and IgG4-RD. A few cases of CSS associated with lachrymal gland swelling have been previously reported $(10,11)$. Takanashi et al. reported that a patient with CSS presented with chronic bilateral dacryoadenitis (11). Additionally, there are two reports of CSS associated with chronic peritonitis $(12,13)$. Unfortunately, these reports did not examine the IgG4 and serum complement levels. The complications associated with CSS are possibly also associated with IgG4-related lesions, as in the present case.

In conclusion, we herein reported a case of CSS concomitant with IgG4-RD. Clinical observations and the serum complement levels are thus considered to be useful for diagnosing IgG4-RD. In addition, there may be a common pathogenesis between CSS and IgG4-RD.

The authors state that they have no Conflict of Interest (COI). 


\section{References}

1. Hamano H, Kawa A, Horiushi A, et al. High serum IgG4 concentrations in patients with sclerosing pancreatitis. $\mathrm{N}$ Eng J Med 344: 732-738, 2001.

2. Yamamoto M, Takahashi H, Suzuki C, et al. Analysis of serum IgG subclass in Churg-Strauss Syndrome-The meaning of Elevated Serum Levels of IgG4. Intern Med 49: 1365-1370, 2010.

3. Vaglio A, Strehl JD, Manger B, et al. IgG4 immune response in Churg-Strauss syndrome. Ann Rheum Dis 11: 390-393, 2011.

4. Masi AT, Hunder GG, Lie JT, et al. The American College of Rheumatology 1990 criteria for the classification of Churg-Strauss syndrome(allergic granulomatous and angiitis). Arthritis Rheum 33: 1094-1100, 1990.

5. JCS Joint Working Group. "Guideline for Management of Vasculitis Syndrome (JCS 2008)." Circ J 75: 474-503, 2011.

6. Takano K, Yamamoto M, Kondo A, Takahashi H, Himi T. A clinical study of olfactory dysfunction in patients with Mikulicz's disease. Auris Nasus Larynx 38: 347-351, 2011.

7. Saeki T, Ito T, Yamazaki H, Imai N, Nishi S. Hypocomplemen- temia of unknown etiology: An opportunity to find cases of IgG4positive multi-organ lymphoproliferative syndrome. Rheumatol Int 30: 99-103, 2009.

8. Yamamoto M, Takahashi H, Shinomura Y. Mikulicz's disease and its extraglandular lesions. Curr Immunol Rev 10: 162-171, 2010.

9. Takahira M, Kawano M, Zen Y, Minato H, Yamada K, Sugiyama K. IgG4-related chronic sclerosing dacryoadenitis. Arch Opthalmol 125: 1575-1578, 2007.

10. Khandwala MA, Vayalambrone D, Ong J, Beigi B. Dacryoadenitis as a presenting feature of Churg-Strauss syndrome. Eye 24: 385386, 2010.

11. Takanashi T, Uchida S, Arita M, Okada M, Kashii S. Orbital inflammatory pseudotumors and ischemic vasculitis in ChurgStrauss syndrome. Ophthalmology 108: 1129-1133, 2001.

12. Fujii K, Hida Y. Churg-Strauss syndrome complicated by chronic periaortitis: A case report and review of the literature. Intern Med 51: 109-112, 2012.

13. Hevier B, Masseau A, Bossard C, et al. Vasa vasoritis of the aorta and fatal myocarditis in fulminant Churg-Strauss syndrome. Rheumatology 47: 1729-1730, 2008.

(C) 2012 The Japanese Society of Internal Medicine http://www.naika.or.jp/imonline/index.html 